\title{
Experimental Study of Arc Motion near Splitter Plates in Low Voltage Switching Devices
}

\author{
Dongkyu Shin, Thomas G. Bull, and John W. McBride
}

\begin{abstract}
Switching performance of a low voltage switching device (LVSD) is determined by limiting current and stopping re-ignition. This is strongly influenced by how the arc moves inside the LVSD quenching chamber during the switching process. Improved switching performance is achieved when the arc quickly enters and stays inside the splitter plates of the LVSD, with the arc attached to the surface of the plates.

This paper studies arc motion around splitter plates in LVSDs and the correlation with arc voltage. Arc motion is investigated using a high-speed optical arc imaging system (AIS). It is observed that the arc fluctuates as it enters the splitter plates, with periods inside the plates followed by sudden re-strikes of the arc roots outside the plates. The AIS system allows the measurement of arc displacement and a correlation with the arc voltage. The results show two phases, with a narrow correlation while the arc in the chamber out of the splitter plates and a distributed correlation while in the splitter plates. Further a new performance parameter the splitter time (ST) ratio is defined. The influence of the ST ratio on the chamber vent design is investigated. It is found that with wider and well distributed vent apertures the ST ratio is higher than with a closed vent, and arc motion on entry to the plates is improved.
\end{abstract}

Index Terms-Arc imaging system (AIS), arc motion, arc voltage, low voltage switching devices, splitter plates, vent condition.

\section{INTRODUCTION}

$\mathrm{T}$ HE switching performance of AC low voltage switching devices (LVSDs) is determined by current limitation and arc re-ignition. The increase in the arc voltage results in significant reduction in the actual current flowing through the device when compared to the prospective current, this is referred to as current limitation. Ideally, the limited arc current is interrupted at the first current zero point; however, it often continues to flow after the current zero point, with the arc re-igniting. Enhanced switching performance is achieved by current limitation without re-ignition [1], [2]. Current limitation and re-ignition are significantly affected by the arc voltage. It is well known that arc motion in the quenching chamber is driven by a combination of Lorentz force and pressure gradient and determines the arc voltage. Therefore, detailed understanding

D. Shin is with the Low Voltage Circuit Breaker Development Department, Hyundai Electric \& Energy system, 17-10, 240 beon-gil, Mabuk-ro, Giheung-gu, Yongin-si, Gyeongi-do 16891, Korea. (e-mail: ds7g14@ gmail.com).

T. G. Bull and J. W. McBride are with the School of Engineering, University of Southampton, Southampton, SO17 1BJ, U.K. of arc motion during LVSD's switching operation is important to improve the design of an LVSD and to enhance switching performance.

Previous empirical investigations have been carried out to study arc motion and its influence on current limitation [3] - [8], re-ignition in an attempt to improve the switching performance [2], [9], [10], or arc splitting process by the splitter plates in the quenching chamber of the LVSD [11].

McBride et al. investigated the influence of several parameters on arc motion (the duration the arc root remains in the contact region) in miniature circuit breakers (MCBs) by recording arc motion through the arc imaging system (AIS) [3] - [5]. It was observed that a high contact opening velocity, wide vent area, closed gap behind the movable contact and iron plate behind the arc runner lead to improved arc motion away from the contact region; conversely, contact material and arc polarity have a minimal influence. Li et al. studied the effects of the arc ignition position and out-gassing materials on arc motion with a simplified chamber geometry without splitter plates [6]. They showed that the larger volume behind the arc, the slower the arc moves with the out-gassing material increasing the arc velocity. Similarly, Yu et al. observed the arc velocity is enhanced by increasing the vent size and chamber width in the simplified quenching chamber [7]. Shin et al. carried out an experimental study on the influence of vent aperture size and distribution on arc motion and interruption performance in LVSDs [8]. They concluded that the arc moves further and more quickly where the vents are larger. In addition, it was shown that well distributed vent apertures increase arc velocity which reduces arcing time.

Chen et al. studied the correlation between arc motion and arc re-ignition in a magnetic contactor using optical fibre arc imaging technology [9]. It was observed that arc motion has a significant influence on probability of arc re-ignition; with higher arc voltage corresponding to better entry of the arc into the splitter plates and therefore a reduced probability of arc re-ignition. Hauer et al. introduced the concept of the exit arc voltage (the arc voltage immediately before the current zero point), as the evaluating parameter for re-ignition [10]. They showed that a higher exit arc voltage results in reduced re-ignition probability in moulded case circuit breakers (MCCBs). Based on the Hauer's study, Shin et al. proposed the arc voltage ratio as a more reliable evaluator of arc re-ignition [2]. By conducting a range of tests on MCBs and MCCBs, it was observed that the arc voltage ratio, defined as the ratio of the recovery voltage to the exit arc voltage, is a reliable 
evaluator for the prediction of re-ignition and a threshold of the voltage ratio exists (at around -2.0), which reliably predicts either successful interruption or instantaneous re-ignition. For example, the probability of instantaneous re-ignition is less than $3 \%$ when the absolute value of exit arc voltage is more than a half of the system voltage. It is noted that the arc voltage has a significant influence on current limitation as well as re-ignition phenomena.

Lindmayer et al. investigated arc motion itself in a simplified quenching chamber with one or two splitter plates [11]. They showed that the arc does not directly enter the splitter plates, but bends and stretches around the plates before the voltage increases enough to form new arc roots on the plate surface.

During the LVSD's switching process, arc voltage fluctuation is observed, which results in deterioration of switching performance. It is assumed that arc voltage fluctuation is related to arc motion, as when the arc is in the splitter plate region arc voltage and the arc shape fluctuate simultaneously [12]; however, there has been little empirical work about the detailed motion of the arc around the splitter plates and the relationship with the arc voltage. This paper focuses on an empirical study of arc motion near the splitter plates in LVSDs, using the AIS [13] to study the correlation between arc motion and voltage, with a particular focus on the interactions in and around the splitter plates. Further to this, the arc voltage and motion around the splitter plates is investigated, while the quenching chamber vent conditions are varied.

\section{EXPERIMENT METHODS AND ARC MOTION ANALYSIS}

The correlation between arc motion and arc voltage is investigated by synchronised recording of ultra-high speed arc imaging data and arc voltage waveform. In addition, several evaluation parameters are proposed to study the effect of vent distribution on arc motion near the splitter plates and switching performance.

\section{A. Arc Imaging Measurement}

\section{1) Flexible Test Apparatus}

Arc motion inside the quenching chamber and splitter plates is investigated by measuring the arc light intensity transmitted through optical fibres. Details of the arc imaging measurement are described in [13]. A flexible test apparatus (FTA) is designed to replicate the quenching chamber of an MCB. This allows the AIS to record arc motion in an MCB under controlled test conditions.

Figure 1 (a) presents the configuration of the FTA including the quenching chamber and operating device (solenoid). Figure 1 (b) shows a 3D image of the chamber with a section through the middle of the splitter plates. The fixed contact is a cathode while the movable contact is an anode. The full open gap between two contacts is $6 \mathrm{~mm}$. Half-cycle current is provided by discharging a capacitor bank. On trigger an event the solenoid arm hits the rotation bar which holds the movable contact and begins to force the contacts apart at a speed of 1.5 $\mathrm{m} / \mathrm{s}$. This begins the arcing period. As current flows through the FTA, the contacts continue to separate and an arc is established between them. After arc ignition, the arc is forced towards the splitter plate region by the Lorentz force and pressure gradient in the chamber. The force from the pressure gradient is mainly determined by the chamber vent opening size and distribution.

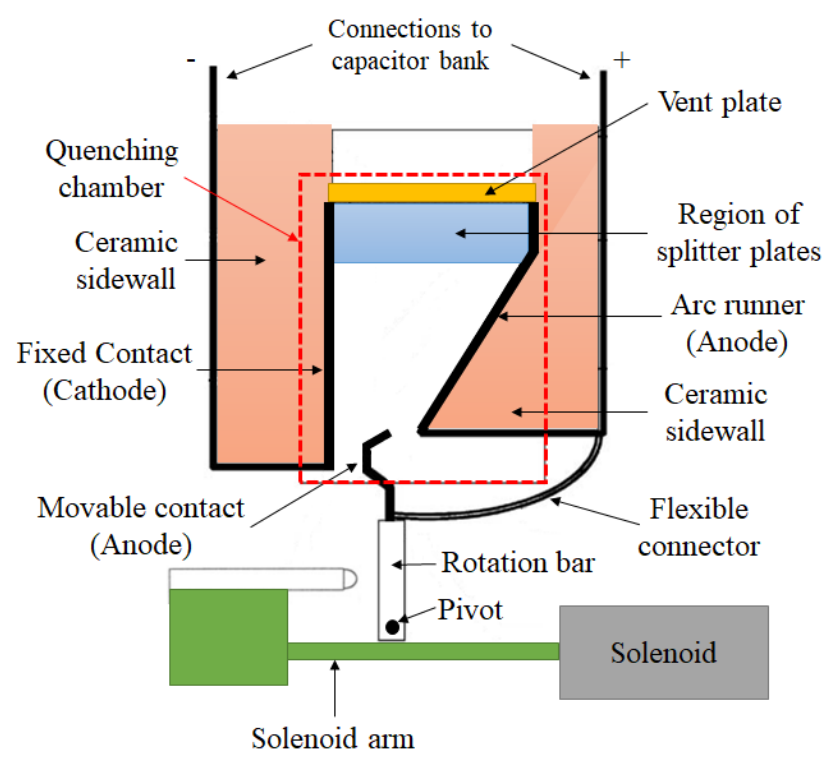

(a) Quenching chamber and operating device (solenoid) of the FTA.

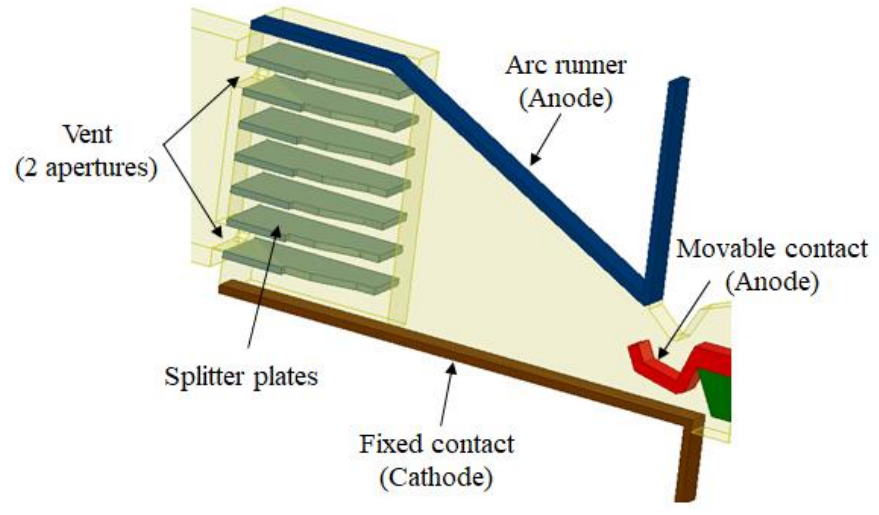

(b) Half symmetric geometry of a quenching chamber in the FTA.

Fig. 1. Configuration of the FTA.

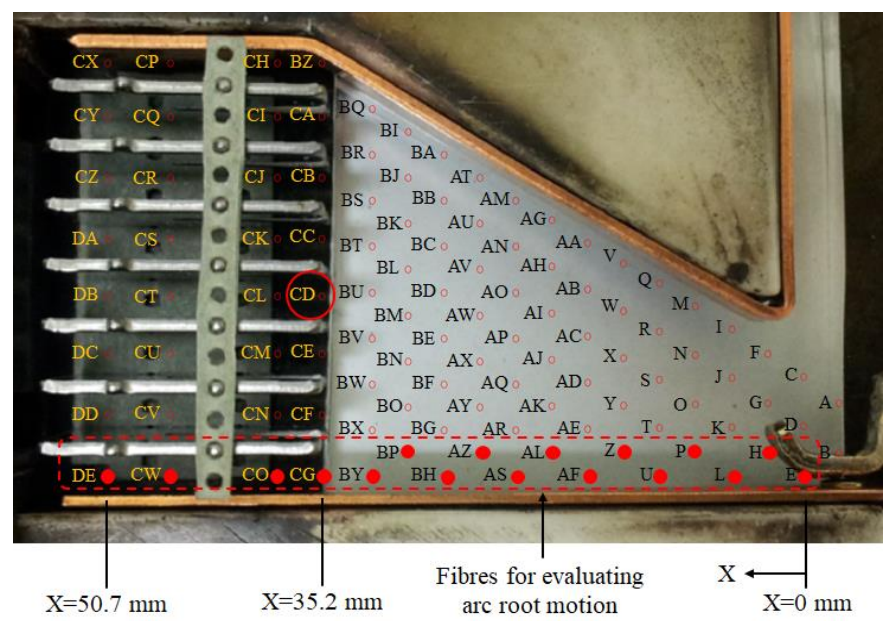

Fig. 2. Fibre locations and names in a quenching chamber and fibres for evaluating cathode arc root motion (horizontal section view), superimposed on a camera image of the arc chamber. 
Figure 2 shows the arrangement of the optical fibres over the quenching chamber of the FTA. Arc light is transmitted through the optical fibres to photodiode sensors with arc light intensity linked to the arc temperature [14]. The position and intensity of the arc light is recorded by the photodiodes to capture the pattern of light at an instant in time (a frame). Frames are captured at $1 \mathrm{MHz}$ during a switching event. This creates a sequence of frames that can then be replayed to show how the arc develops and moves through the quenching chamber.

\section{2) Arc Imaging Display}

The arc visual analysis software is used here to link the light intensity together with coloured contours to show arc motion. There are two modes for the colour contours: fixed or dynamic threshold [12]. The fixed threshold mode uses the maximum light intensity for the whole data set to set the colour scale and it is suitable for an overview of the arc process. In the dynamic threshold mode, the maximum light intensity in each frame is used as the highest value for the colour scale, so it is good at displaying the arc when light intensity is low. A 7 colour scale of contours is used to show the arc light intensity. In this study the light intensity is relatively low and the light intensity contours levels used are also set relatively low to provide optimal images of the arc. Low contour levels highlight the central region of the arc event, for imaging only and does not affect the calculation of the cathode arc root trajectory. The colour scale representing light intensity is shown in Fig. 3.

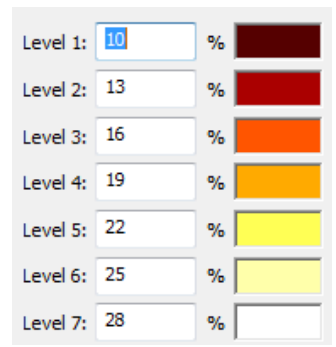

Fig. 3. Contour levels set as [10\%, $13 \%, 16 \%, 19 \%, 22 \%, 25 \%, 28 \%]$.

TABLE I

EXPERIMENT CONDITIONS.

\begin{tabular}{|c|c|c|c|c|}
\hline \multirow{2}{*}{ Experiment } & \multicolumn{4}{|c|}{ Experiment conditions } \\
\hline & $\begin{array}{l}\text { Splitter } \\
\text { Plates }\end{array}$ & $\begin{array}{c}\text { Current } \\
\left(\mathrm{A}_{\text {peak }}\right)\end{array}$ & $\begin{array}{c}\text { Vent } \\
\text { Apertures }\end{array}$ & Vent Opening (\%) \\
\hline $\begin{array}{c}\text { 1. Arc } \\
\text { Motion on } \\
\text { Arc Voltage }\end{array}$ & \multirow{6}{*}{$\begin{array}{l}7 \text { iron } \\
\text { plates }\end{array}$} & \multirow{6}{*}{1800} & 2 & Partially Open (23\%) \\
\hline \multirow{3}{*}{ 2. Vent Area } & & & \multirow{3}{*}{2} & Fully Closed (0\%) \\
\hline & & & & Partially Open (23\%) \\
\hline & & & & Fully Open (100\%) \\
\hline \multirow{2}{*}{$\begin{array}{c}\text { 3. Vent } \\
\text { Distribution }\end{array}$} & & & 2 & \multirow{2}{*}{ Partially Open (23\%) } \\
\hline & & & 12 & \\
\hline
\end{tabular}

\section{3) Cathode Arc Root Trajectory}

Arc root trajectory at the cathode is obtained by the centre of intensity method,

$$
x_{C R}=\frac{\sum I_{i} X_{i}}{\sum I_{i}},
$$

where, $x_{C R}$ is the centre of light intensity, $I_{i}$ is the light intensity at a time for the fibre at position $X_{i}$ next to the cathode surface as shown in Fig. 2 [13]. The cathode trajectory presents the position of cathode root in the $\mathrm{X}$ direction (from the ignition point to the region of the splitter plates). To remove the influence of stray light when calculating the arc trajectory, a minimum light intensity threshold filter is first applied.

\section{B. Experimental Conditions}

Table I summarises conditions for each test. The switching tests used to investigate the correlation between arc motion and arc voltage are carried out at $1800 \mathrm{~A}_{\text {peak }}$ half-cycle current using 2 vent apertures (see Fig. 4 (a)) with $23 \%$ of the venting area opened. To study the effect of vent size three vent conditions are investigated: fully closed, partially open and fully open $(0 \%, 23 \%$, and $100 \%$ of the venting area opened). The effect of the vent distribution is tested using two vent configurations ( 2 and 12 apertures) as shown in Fig. 4. Both aperture designs have the same total area and are tested with the same percentage opened area (23\% opening of the total vent area). Figure 5 shows the iron splitter plate used in the quenching chamber and the names and locations of the optical fibre inside the splitter plate region. In Fig. 5, the 4 fibre positions correspond to the gap between the cathode fixed contact and the nearest plate to the cathode. It should be noted that the optical fibre positions only allow for the evaluation of the $\mathrm{X}$ position of the arc, and do not indicate

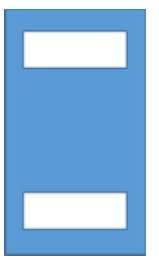

(a) 2 apertures vent

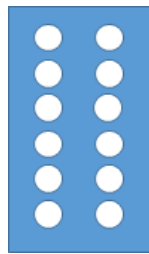

(b) 12 apertures vent
Fig. 4. Two types of vent distribution tested, both with $23 \%$ vent area open.

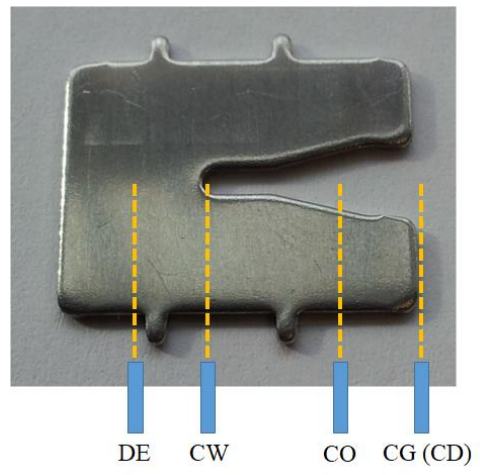

Fig. 5. Geometry of splitter plate and optical fibre locations in the gap between the cathode fixed contact and the nearest plate. 
the location of the arc root across the face of the plate. Iron is widely used as the splitter plate in LVSDs because the magnetic properties improve arc motion towards the splitter plates. 1800 A peak current flows through the FTA during capacitor bank discharge from $200 \mathrm{~V}$. Each current interruption test is repeated three times. After three tests, the splitter plates, arc runner, fixed and movable contacts are replaced for the next repeat tests.

\section{Evaluation of Arc motion and Switching Performance}

To evaluate the effects of current, splitter plate, and vent condition on arc motion and LVSD switching performance, 6 evaluation parameters are introduced and defined by (2) - (7) below

$$
\begin{gathered}
X_{\text {aver }}=\frac{\int_{t_{1}}^{t_{2}} x(t) d t}{t_{2}-t_{1}}, \\
V e l_{\text {aver }}=\frac{X_{\text {aver }}}{t_{\text {arcing }}}, \\
U_{\text {aver }}=\frac{\int_{t_{0}}^{t_{2}} U_{\text {arc }}(t) d t}{t_{2}-t_{0}}, \\
I^{2} t=\int_{t_{0}}^{t_{2}} I^{2}(t) d t, \\
t_{\text {arcing }}=t_{2}-t_{0},
\end{gathered}
$$

$$
\text { Splitter Time }(S T) \text { ratio }=\frac{t_{x_{C R}>35.2 \mathrm{~mm}}}{t_{\text {arcing }}},
$$

where, $X_{\text {aver }}$ is the average displacement of the cathode root during the period of arc light existence from the start of arc light $\left(t_{1}\right)$ to the arc extinguish moment $\left(t_{2}\right), V_{e} l_{\text {aver }}$ is the average velocity of the arc during the period of arc light existence, $U_{\text {aver }}$ is the average arc voltage during the arcing time, $I^{2} t$ is the net-through energy, $t_{\text {arcing }}$ is the arcing time, $x$ is the cathode arc root trajectory (or the centre of light intensity), $U_{\text {arc }}$ is the arc voltage, $I$ is the current. The time periods are shown in Fig. 6, where $t_{0}$ is the arc ignition time (or the contact separation time), $t_{1}$ is the starting time of the cathode root motion and $t_{2}$ is the time when the arc extinguishes. The Splitter Time ratio ( $S T$ ratio) is a new parameter defined as the ratio of $t_{x C R}>35.2 \mathrm{~mm}$ to $t_{\text {arcing. }} t_{x C R}>35.2 \mathrm{~mm}$ refers to the time duration when the arc root trajectory is greater than $35.2 \mathrm{~mm}$; therefore, it quantifies how well the arc stays in the splitter plate region during switching process. Enhanced switching performance during the interruption process is achieved with the arc stable inside the splitter plates and with the arc itself attached to the surface of the plates. The $S T$ ratio represents the ratio of arc time inside the splitter plates to the total arcing time; therefore, it describes the splitter plate performance significantly influencing the current limitation and overall switching performance of LVSDs. In general, the higher the $S T$ ratio (the better the splitter plate performance), the better the current limitation and the better the switching performance.

\section{RESULTS AND DISCUSSION}

\section{A. Experiment 1: Correlation between Arc Motion and Arc Voltage}

1) Overview

Figure 6 presents the arc voltage and the cathode arc root trajectory, with the corresponding arc imaging data shown in Fig. 7. The test is carried out with parameters in Table I: partially open vent (see Fig. 4 (a)) and 1800 A peak current. The 3 tests showed a high degree of repeatability in overall parameters. The data shown here is for the experiment with the closest arcing time to the average of the 3 tests.

Synchronisation is observed between the drops of arc voltage and the apparent backward arc steps; when the cathode root instantly jumps out of the splitter plate region, the arc voltage falls. Figure 7 shows the arc images at instants in time corresponding to events (a) - (p) on the arc voltage and cathode root trajectory in Fig. 6. The arc images are shown in dynamic threshold mode and with the light intensity representing by colour as shown in Fig. 3. After ignition, the arc elongates as the gap between the movable and fixed contact increases (see Fig. 7 (a) and (b)). The anode arc root jumps from the movable contact to the arc runner at around $4.0 \mathrm{~ms}$ (see Fig. 7 (b)). The arc gradually moves towards the splitter plates and cathode root enters the splitter plates with corresponding increase in the arc voltage; however, the cathode arc root suddenly backward steps by $8 \mathrm{~mm}$ at $4.6 \mathrm{~ms}$ (see Fig. 7 (c) and (d)) and there is another significant backward step of about $20 \mathrm{~mm}$ at $4.85 \mathrm{~ms}$ (see Fig. 7 (e) (h)), with corresponding drops in arc voltage. The image Fig. 7 (f) shows the important point where there are two cathode arc roots, one at the base of the splitter plates and one in the contact region. It is important to note that the photographic background images used in Fig. 7 is a fixed single camera image frame with the contacts closed. It is used to show the position of the features only. At Fig. 7 (f) it is likely that the moving contact will have re-bounded and that the contact gap will have reduced, leading to a relatively low conductivity path for the gas discharge. This backwards stepping occurs throughout the experiment, with no evidence of a uniform motion of the cathode arc root which is shown when $\mathrm{X}$ is increasing.

After this, the arc is again forced into the splitter plates by the gas pressure gradient and Lorentz force, but does not remain continuously within the splitter plates with repeat arc back-stepping (see Fig. 7 (i) (p)). Even though the arc fully enters the splitter plates, it steps back and the arc is later seen outside the splitter plates (see Fig. $7(\mathrm{~m}) \sim(\mathrm{p})$ ). The arc is fully in the plates at Fig. $7(\mathrm{~m})$ and briefly stable before $26 \mu$ s later a new arc root forms outside the splitter plate region and a new arc column region forms at position Fig. 7 (n). The MHz frame rate captures the details of the re-strike event with the co-existence of two cathode arc roots, one inside the splitter plates and one in the arc chamber out of plates. The images show there is a period when both regions Fig. 7 (m) and Fig. 7 (n) are conductive, as evidences in Fig. 7 (n). Similarly, the re-strike phenomenon is observed between Fig. 7 (o) and (p). 


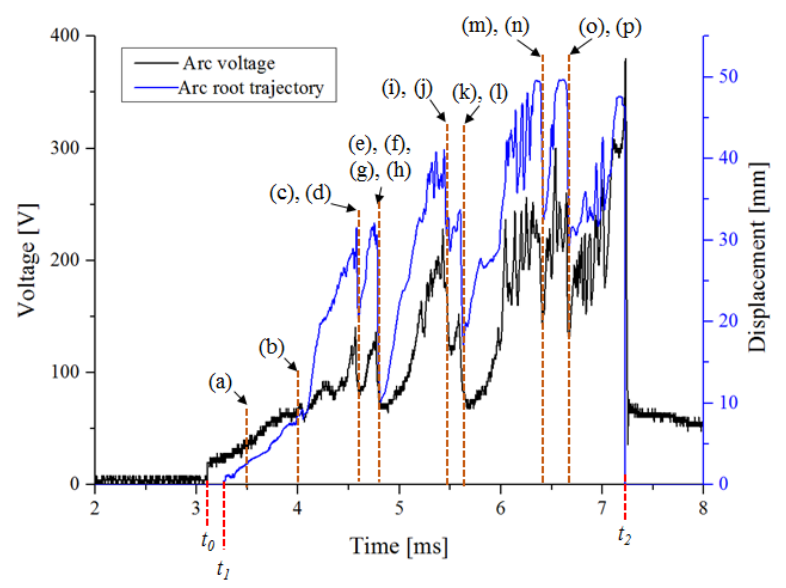

Fig. 6. Arc voltage and cathode root trajectory waveforms; $t_{0}$ is the arc ignition time (or the contact separation time), $t_{l}$ is the starting time of the cathode root motion and $t_{2}$ is the time when the arc extinguishes.

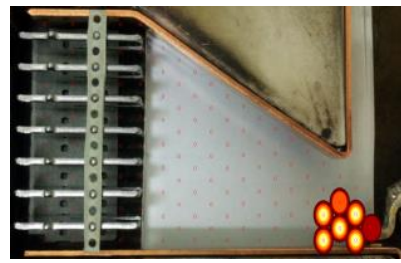

(a) $3.500 \mathrm{~ms}$

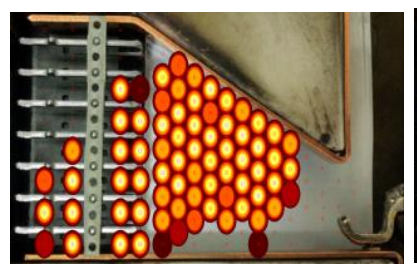

(c) $4.578 \mathrm{~ms}$

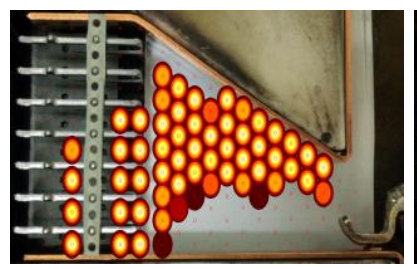

(e) $4.782 \mathrm{~ms}$

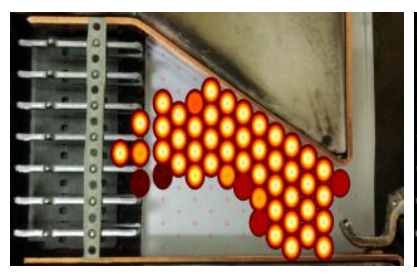

(g) $4.807 \mathrm{~ms}$

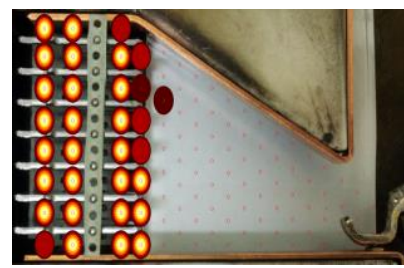

(i) $5.433 \mathrm{~ms}$

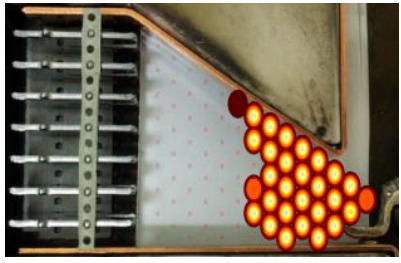

(b) $4.000 \mathrm{~ms}$

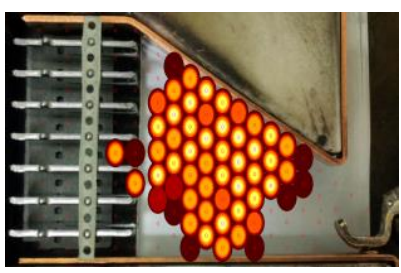

(d) $4.600 \mathrm{~ms}$

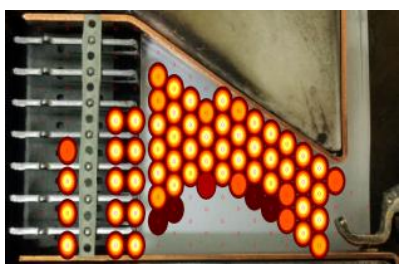

(f) $4.785 \mathrm{~ms}$

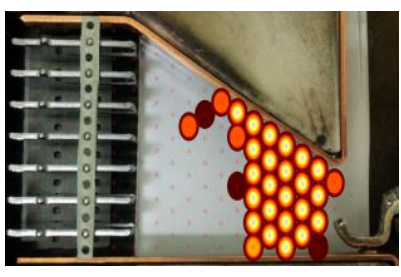

(h) $4.850 \mathrm{~ms}$

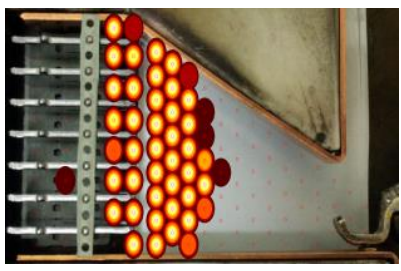

(j) $5.500 \mathrm{~ms}$

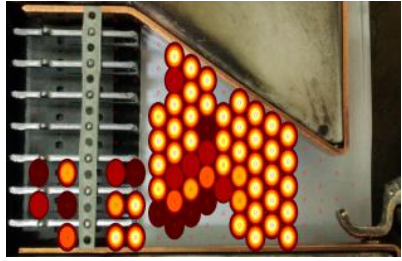

(k) $5.619 \mathrm{~ms}$

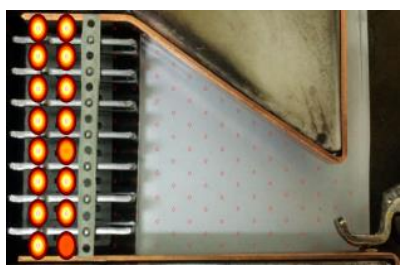

(m) $6.392 \mathrm{~ms}$

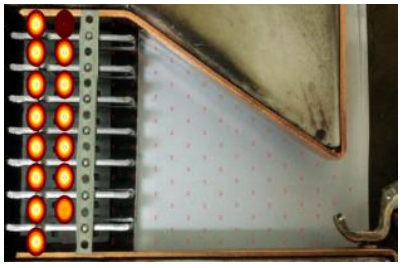

(o) $6.651 \mathrm{~ms}$

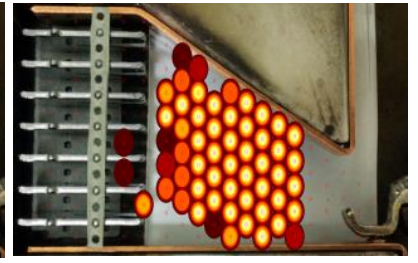

(1) $5.632 \mathrm{~ms}$

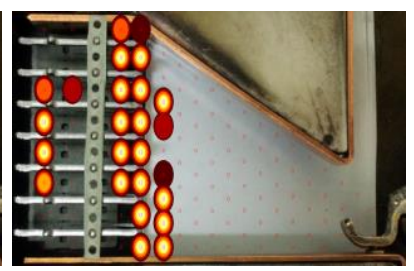

(n) $6.418 \mathrm{~ms}$

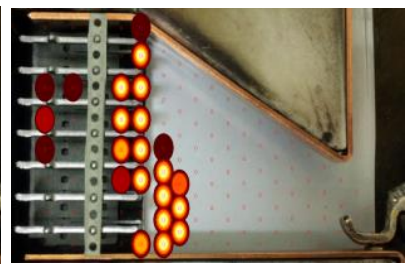

(p) $6.671 \mathrm{~ms}$
Fig. 7. Arc images during interruption process: each image corresponds to (a) (p) instants in Fig. 6: dynamic threshold mode and contour level of $[10 \%, 13 \%$, $16 \%, 19 \%, 22 \%, 25 \%, 28 \%$ ].

\section{2) High Frequency Events}

As well as the low frequency voltage fluctuations, there are high frequency fluctuations (microscopic fluctuations of arc voltage) observed when the arc is near the splitter plates after $6.0 \mathrm{~ms}$. The whole arc motion and splitting phenomena are significantly influenced by the arc roots and arc column positions; therefore, the arc voltage fluctuations are caused by the fluctuations in the arc roots and arc column positions. To show the interaction between the entire arc motion (arc root and column) and the microscopic voltage fluctuation, the arc voltage and cathode root trajectory waveforms are magnified and shown in Fig. 8. It is seen that high frequency arc voltage fluctuations are linked to the fluctuations of cathode root position in region B (corresponding arc root position between $35.2 \mathrm{~mm}$ and $50 \mathrm{~mm}$ ). However, there are some regions (region $\mathrm{A}, \mathrm{C}$, and $\mathrm{D}$ ) where the arc voltage fluctuations are not correlated with the cathode root position. For example, the arc voltage is significantly fluctuating in region $\mathrm{C}$ prior to position (o) at $6.6 \mathrm{~ms}$ where the arc root is fixed at $50 \mathrm{~mm}$. These high frequency arc fluctuations are explained by the arc column position.

A detailed inspection of the light intensity transmitted by a single optical fibre shows the evidence of the interaction between arc column position and the high frequency fluctuation of arc voltage. The arc voltage graph and light intensity of the fibre $\mathrm{CD}$ are presented in Fig. 9. The fibre $\mathrm{CD}$ is shown by a red circle in Fig. 2 and corresponds to the same X position as CG in Fig. 5. A clear correlation between the arc voltage and the light intensity at fibre $\mathrm{CD}$ at a high frequency is observed in region $\mathrm{A}$, $\mathrm{B}$, and $\mathrm{D}$ in Fig 9. In general, when the arc column comes out 


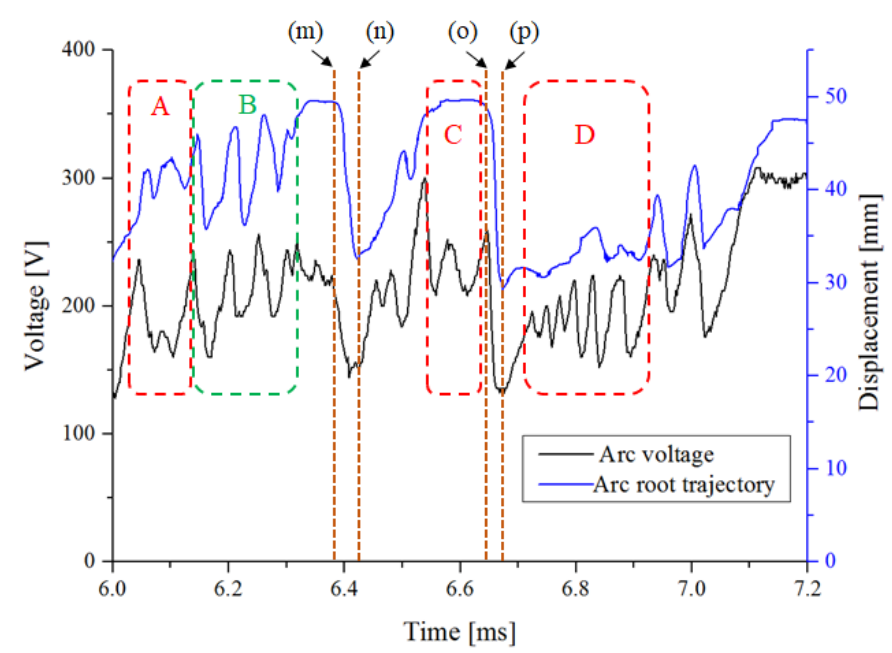

Fig. 8. Arc voltage and cathode root trajectory waveforms from $6.0 \mathrm{~ms}$ to 7.2 ms.

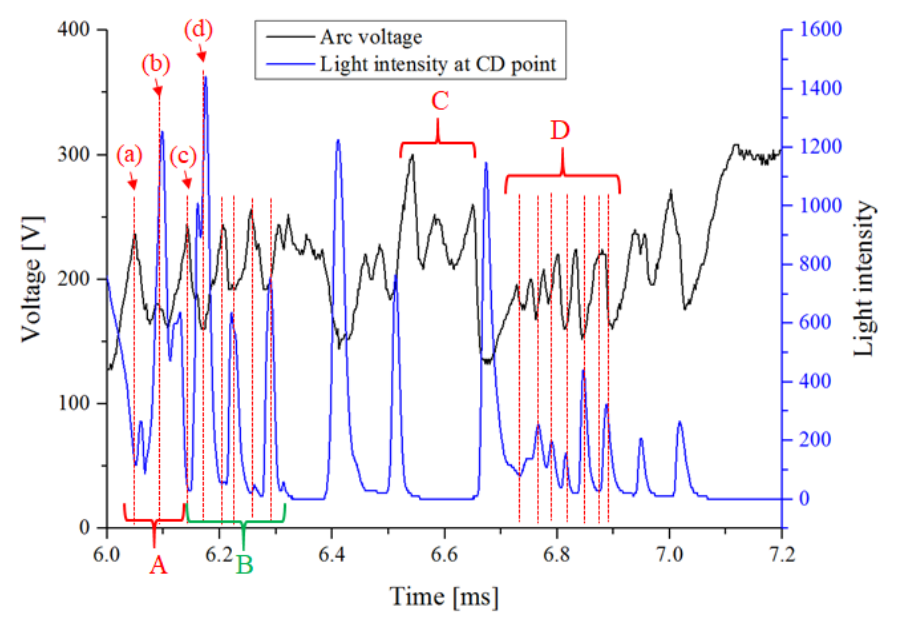

Fig. 9. Arc voltage and light intensity on fibre CD.

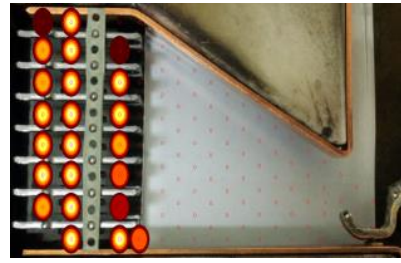

(a) $6.052 \mathrm{~ms}(236 \mathrm{~V})$

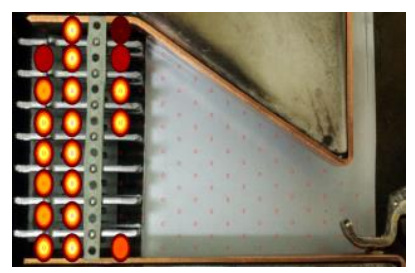

(c) $6.145 \mathrm{~ms}(244 \mathrm{~V})$

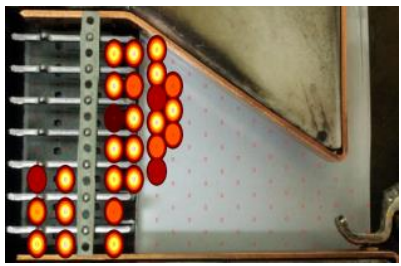

(b) $6.095 \mathrm{~ms}(176.0 \mathrm{~V})$

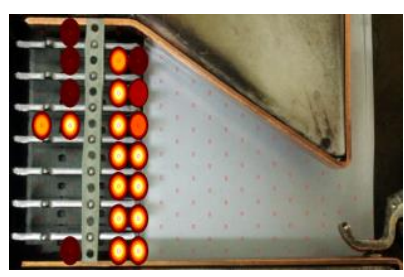

(d) $6.177 \mathrm{~ms}(160 \mathrm{~V})$
Fig. 10. Arc images at (a) (d) instants in Fig. 9; the value in the round bracket is the arc voltage, arc images are diplayed with the fixed threshold mode and contour level of $[10 \%, 13 \%, 16 \%, 19 \%, 22 \%, 25 \%, 28 \%]$.

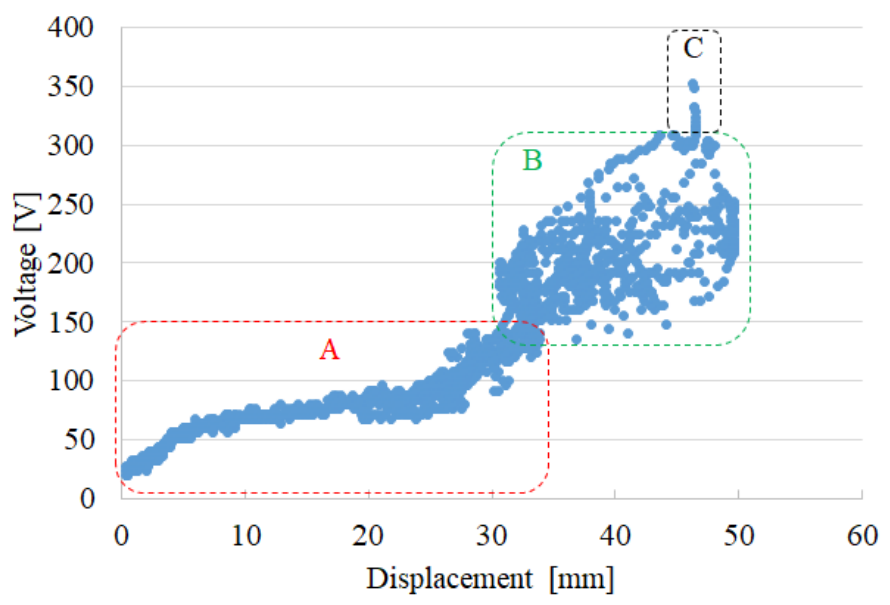

Fig. 11. Correlation between the arc voltage and cathode root trajectory during the arcing time.

from the splitter plates the arc voltage drops. Figure 10 shows the arc images at (a) (d) instance in Fig. 9 where the arc voltage and light intensity of fibre $\mathrm{CD}$ have a local peak between $6.00 \mathrm{~ms}$ and $6.2 \mathrm{~ms}$. Figure 10 (a) and (b) show arc motion in region A while Fig. 10 (c) and (d) are a part of region B. The arc almost fully enters the splitter plates at $6.052 \mathrm{~ms}$ with an arc voltage at $236 \mathrm{~V}$ (see Fig. 10 (a)); however, shortly after the anode arc root suddenly jumps backwards with a drop of $60 \mathrm{~V}$ arc voltage. The upper half part of arc (including the fibre $\mathrm{CD}$ position) is observed at the front of the splitter plates whereas the cathode arc root remains inside the plate region (see Fig. 10 (b)); therefore, the arc voltage fluctuation is not correlated with the cathode root position in region A. There is another backward step of arc motion between Fig. 10 (c) and (d) with an arc voltage drop of $84 \mathrm{~V}$. Unlike Fig. 10 (b), the entire arc (cathode/anode roots and column) steps backwards in Fig. 10. (d); therefore, the arc voltage fluctuation is related to both cathode root position and arc column positon (light intensity of the fibre CD) in region B. This instability of arc motion (arc roots and arc column positions) and voltage near and inside the splitter plate region repeats a number of times before the arc stabilises at around $7.15 \mathrm{~ms}$, with arc voltage relatively stable for the remainder of the switching event. These results provide an insight into complexities that would do difficult to model with conventional arc modelling methods [15].

In Fig. 8 and 9, there are some regions where the arc voltage is not correlated with the cathode root displacement or light intensity from the single optical fibre $\mathrm{CD}$. For example, from $6.55 \mathrm{~ms}$ to $6.65 \mathrm{~ms}$ (region $\mathrm{C}$ in Fig. 8 and 9) there is no correlation between the arc voltage and cathode root or between the arc voltage and intensity of the fibre CD. It is noted that analysis of a single fibre position light intensity or arc root position does not describe the behaviour of the entire arc.

\section{3) Correlation between Arc Voltage and Cathode Root Trajectory}

Figure 11 shows the correlation between the arc voltage and cathode root trajectory during the arcing time. The graph is replotted based on Fig. 6, which has the displacement of the 
cathode root trajectory in the $\mathrm{X}$ axis and the arc voltage in the $\mathrm{Y}$ axis. Each point represents the cathode root position and arc voltage at a specific instant. Three regions $(\mathrm{A}, \mathrm{B}$, and $\mathrm{C})$ are observed in Fig. 11 and each region has a distinct pattern. It is shown that in region A when the arc is in the arc chamber out of the splitter plates the voltage and cathode root displacement are highly correlated, while in region B over $30 \mathrm{~mm}$ the arc is near or in the splitter plates and for a given arc root position the arc voltage can have a range of values. For example, at $40 \mathrm{~mm}$ the arc voltage can be between $150 \mathrm{~V}$ and $280 \mathrm{~V}$. There is a bounded area between $140 \mathrm{~V}$ and $300 \mathrm{~V}$ in region $\mathrm{B}$. The upper bounding curve of region $\mathrm{B}$ is a line, while at the $46 \mathrm{~mm}$ position (region $\mathrm{C}$ ) there is the highest arc voltage outside the bounded area B. The arc voltage increases from $316 \mathrm{~V}$ to $352 \mathrm{~V}$ as the current decreases from $90 \mathrm{~A}$ to $0 \mathrm{~A}$ in the region $\mathrm{C}$, prior to arc extinction.

Two features can be noted here. Firstly, there is a narrow correlation between the arc voltage and cathode root trajectory while the arc is in the chamber out of the splitter plates because the cathode root position has a great influence on the entire arc length that determines the arc voltage. Secondly, a distributed correlation is observed while the arc is near or within the splitter plate region because the cathode root position has the lack of information about the entire arc motion and splitting phenomena. There is a significant large bounded area of arc

TABLE II

EXPERIMENTAL ResUlts ASSOCIATED With VeNT Size.

\begin{tabular}{|c|c|c|c|}
\hline & Closed & Partially open & Fully open \\
\hline$X_{\text {aver }}[\mathrm{mm}]$ & 23.2 & 25.6 & 34.5 \\
\hline$V_{e l}[\mathrm{mver}[\mathrm{m} / \mathrm{s}]$ & 4.57 & 6.38 & 9.43 \\
\hline$U_{\text {aver }}[\mathrm{V}]$ & 95.3 & 124.6 & 143.3 \\
\hline$I^{2} t\left[\mathrm{~A}^{2} \mathrm{~s}\right]$ & 7501 & 6974 & 5788 \\
\hline$t_{\text {arcing }}[\mathrm{ms}]$ & 5.08 & 4.01 & 3.66 \\
\hline ST ratio & 0.12 & 0.27 & 0.55 \\
\hline
\end{tabular}

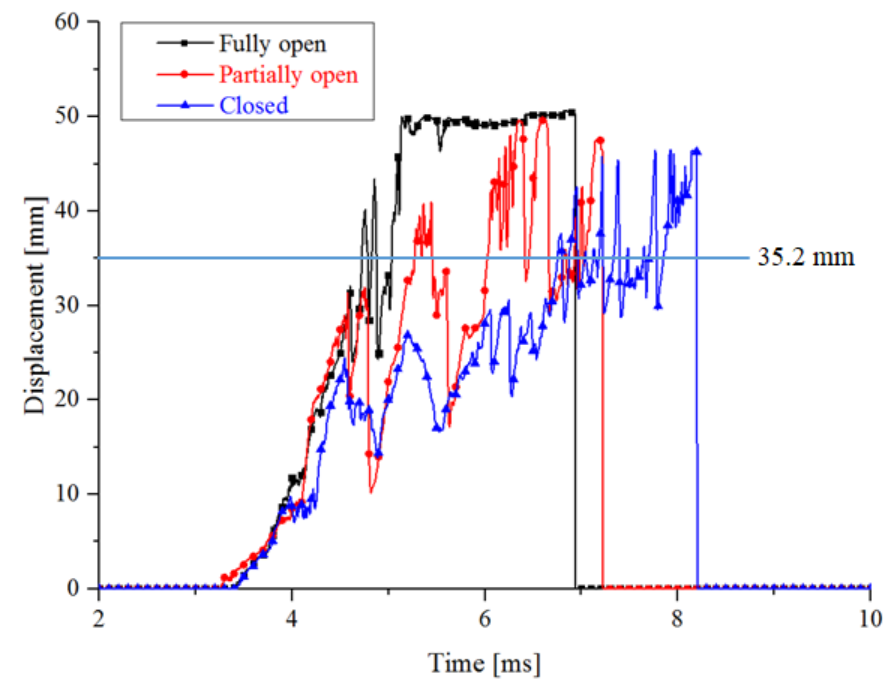

Fig. 12. Cathode root trajectory waveforms depending on vent size; a single test result.

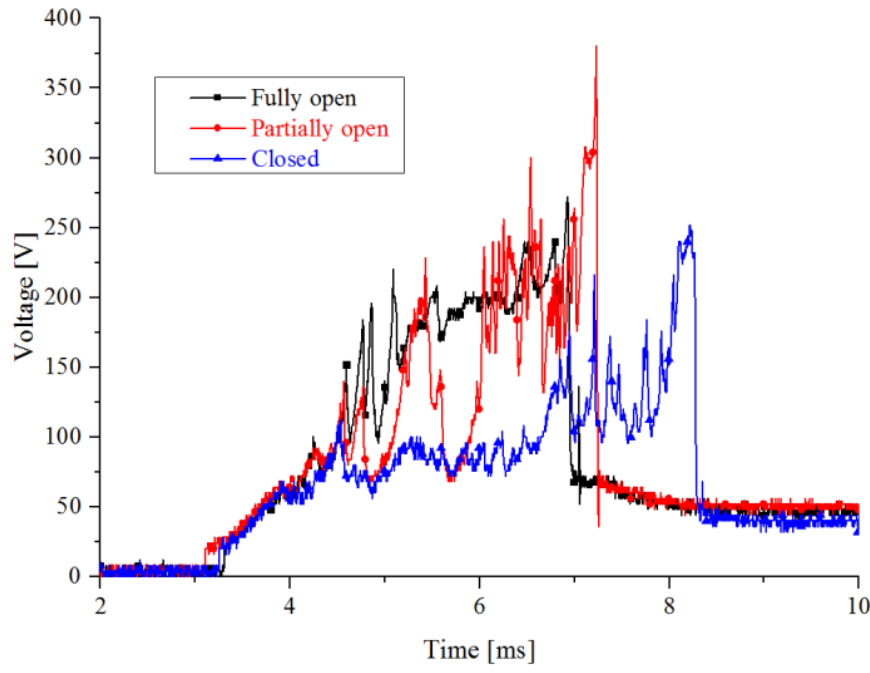

Fig. 13. Arc voltage waveforms depending on vent size; a single test result.

voltage when the arc is near the splitter plates, which can be explained by the high frequency voltage fluctuation influenced by the entire arc motion (arc roots and column positions) instability in the splitter plate region.

\section{B. Experiment 2: Effect of Vent Size on Arc Motion}

Arc motion and switching performance dependence on the vent size are investigated by comparing 6 evaluation parameters defined by (2) - (7) and the cathode root trajectories. Table II presents the measured effects of the vent size on the evaluation parameters obtained from the average of 3 repeat switching tests.

With a wider vent the average displacement of the cathode root $\left(X_{\text {aver }}\right)$, average velocity $\left(\right.$ Vel $\left._{\text {aver }}\right)$, average arc voltage $\left(U_{\text {aver }}\right)$ and $S T$ ratio increase, while net-through energy $\left(I^{2} t\right)$ and arcing time $\left(t_{\text {arcing }}\right)$ decrease; all benefit the switching performance. It is also noted that the arc moves more quickly towards the splitter plates and stays inside the splitter plates with less backward steps with increased vent area.

Figure 12 and 13 show the cathode root trajectory and arc voltage waveforms plotted against time for the three vent size conditions. Each plot is a single test result (not an average value). In the fully opened vent condition the arc quickly reaches the splitter plates and remains relatively stable at the region of splitter plates when compared to the partially open or closed cases; therefore, a relatively high and stable arc voltage is observed in the fully opened vent condition, which leads to the highest average arc voltage $(143.3 \mathrm{~V})$. The $S T$ ratio shows that the arc is in the splitter plates for $55 \%$ of the arcing time. In contrast in the partially open vent condition, the arc remains inside the splitter plate region for only $27 \%$ of the arcing time with notable backward arc steps and arc voltage drops. At the end of the arcing time, the arc voltage in the partially open vent condition is higher than that in the fully opened case due to a higher pressure inside the chamber by a smaller vent area. In the fully closed vent condition the arc hardly enters the splitter plates, and the lowest arc velocity $(4.57 \mathrm{~m} / \mathrm{s})$ and ST ratio $(12 \%)$ are observed. This leads to the lowest average arc voltage (95.3 $\mathrm{V})$ and poor current limitation during the switching process. 
The $S T$ ratio is identified as a key parameter in understanding the splitter plate performance. The $S T$ ratio greatly affects $U_{\text {aver }}$ since the multiple split arcs in the splitter plate region generate a higher arc voltage; therefore, $I^{2} t$ and $t_{\text {arcing }}$ decrease due to enhanced current limitation in the case of a higher ST ratio.

\section{Experiment 3: Effect of Vent Distribution on Arc Motion}

Table III shows the effect of vent aperture distribution on arc motion during the switching process. It is seen that well distributed vent achieves greater movement of the arc towards the splitter plates and remains in the splitter plate region more consistently with decreased arc back-steps. The arc moves more smoothly towards the splitter plates and better enters them in the case of the 12 apertures when compared to the 2 apertures case ( $X_{\text {aver }}$ and $V e l_{\text {aver }}$ of 12 apertures are higher than those of 2 apertures). The arc voltage is also enhanced in the 12 aperture case. This is attributed to smoother motion of the arc and its consistent entry to the splitter plates ( $V_{\text {aver }}$ of 12 apertures is higher by $8.3 \%$ than that of 2 apertures). The net-through energy and arcing time decrease due to improved current limitation $\left(I^{2} t\right.$ and $t_{\text {arcing }}$ of 12 apertures are lower by $5.2 \%$ and $6.7 \%$, respectively, than those of 2 apertures). The tendency of arc remaining in the splitter plates is more pronounced in the 12 apertures cases (ST ratio of 12 apertures is greater by $37 \%$ than that of 2 apertures).

TABLE III

EXPERIMENTAL RESULTS ASSOCIATED WITH VENT DISTRIBUTION.

\begin{tabular}{|c|c|c|}
\hline & 2 apertures & 12 apertures \\
\hline$X_{\text {aver }}[\mathrm{mm}]$ & 25.6 & 28.9 \\
\hline$V e l_{\text {aver }}[\mathrm{m} / \mathrm{s}]$ & 6.38 & 7.73 \\
\hline$V_{\text {aver }}[\mathrm{V}]$ & 124.6 & 134.9 \\
\hline$I^{2} t\left[\mathrm{~A}^{2} \mathrm{~s}\right]$ & 6974 & 6615 \\
\hline$t_{\text {arcing }}[\mathrm{ms}]$ & 4.01 & 3.74 \\
\hline$S T$ ratio & 0.27 & 0.37 \\
\hline
\end{tabular}

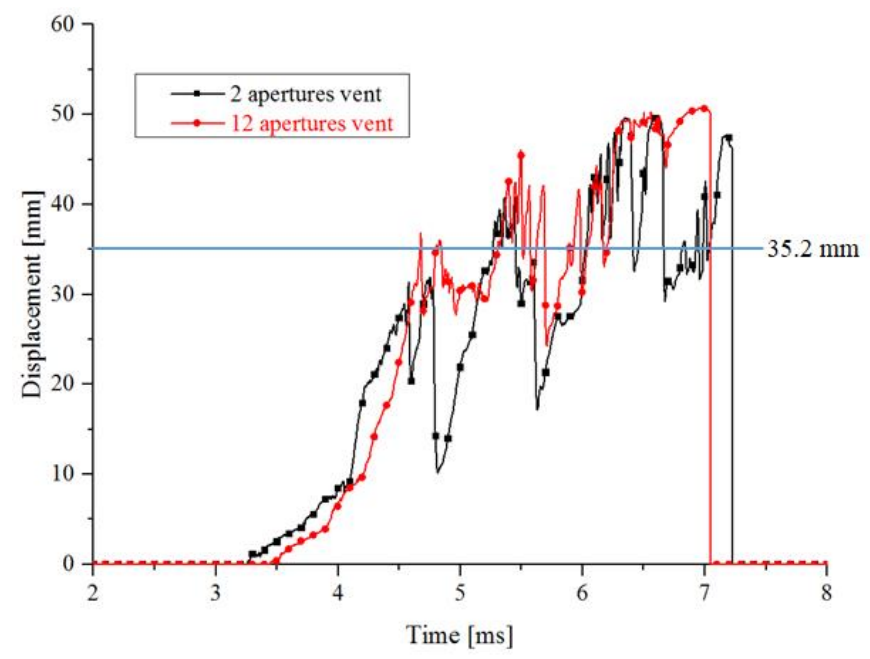

Fig. 14. Cathode root trajectory waveforms depending on vent distribution; a single test result.

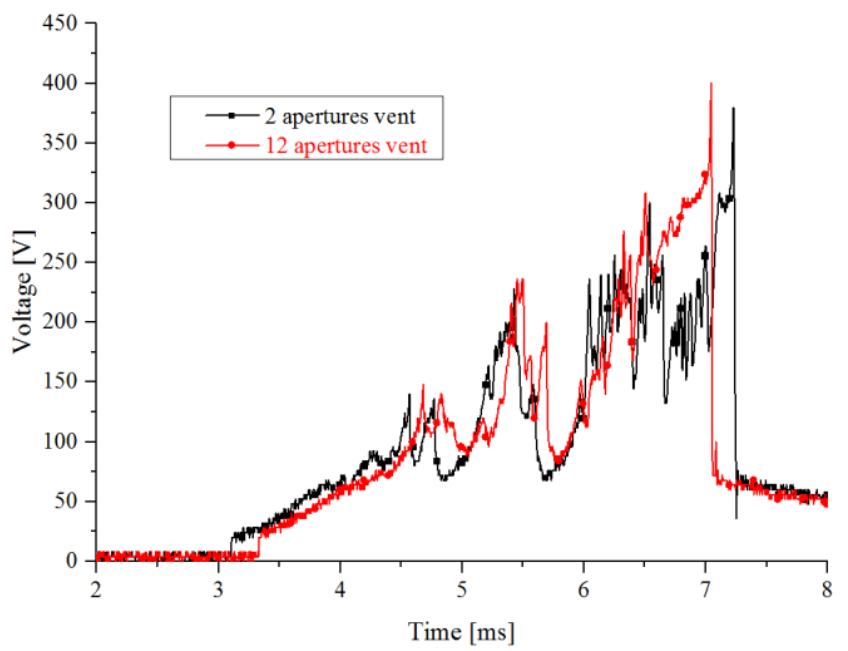

Fig. 15. Arc voltage waveforms depending on vent distribution; a single test result.

Figure 14 and 15 show the cathode root trajectory and arc voltage waveforms for the 2 and 12 apertures cases. Much less fluctuation and more stable behaviour of the arc voltage and arc motion in the plates region are observed in the well distributed 12 vent case. A significant backward arc step (defined as the arc retreating from the splitter plates by more than $15 \mathrm{~mm}$ ) is observed only once at around $5.7 \mathrm{~ms}$ in the 12 aperture vent, resulting in the arc voltage drop of $100 \mathrm{~V}$. In the 2 aperture vent more back steps are observed with 4 detected at around $4.8 \mathrm{~ms}$ (with the arc voltage drop of $68 \mathrm{~V}$ ), $5.6 \mathrm{~ms}$ (with the arc voltage drop of $84 \mathrm{~V}$ ), $6.4 \mathrm{~ms}$ (with the arc voltage drop of $80 \mathrm{~V}$ ), and $6.7 \mathrm{~ms}$ (with the arc voltage drop of $128 \mathrm{~V}$ ). After $6.2 \mathrm{~ms}$ the arc remains stably within the splitter plates in the 12 vent case; therefore, there is no significant drops of the arc voltage. In contrast for the 2 aperture vent there is no period where arc motion is stable in the splitter plates.

\section{D.Discussion}

It is observed that the arc motion instability (repeated forward/backward step of arc roots and column) near or within the splitter plate region leads to the high frequency fluctuations of the arc voltage. The arc motion instability is caused by complex interaction of electromagnetic and pressure forces and the cathode arc root preference to a given location. A threshold energy is required to form and sustain a new arc root on the surface of the splitter plate. The arc root region has a much lower conductance (much higher resistance) than the arc column. If there is sufficient Lorentz force or pressure gradient applied to the arc, it enters the splitter plates leading to new arc roots formed inside the splitter plates with a consequent increase in the arc voltage. However, the hot gas remains in the quenching chamber and in front of the splitter plates. The conductance of the hot gas in front of the splitter plates can be higher than that of the newly formed arc (which consists of new arc roots and column) inside the splitter. In this situation, a new current path appears outside of (in front of) the splitter plates and replaces the original arc position. This phenomenon causes the instability of the arc position (repeated forward motion and backward step) near or within the splitter plates, which is 


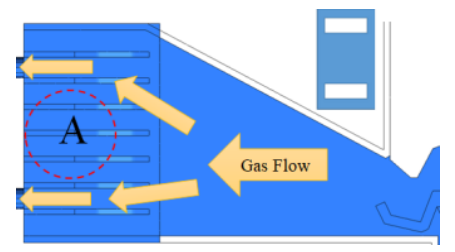

(a) 2 apertures vent

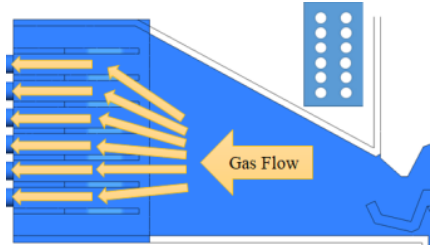

(b) 12 apertures vent
Fig. 16. Comparison of gas flows in the case of 2 apertures and 12 apertures vent.

related to the high frequency fluctuation of the arc voltage.

In the LVSDs, the arc voltage is highly dependent on the cathode root trajectory before the arc reaches the splitter plates; however, the arc voltage is not correlated with the cathode root position when the arc is near or within the splitter plate region because the cathode root position has the lack of information about the whole of arc motion and arc splitting phenomena. The motion instability in a small part of arc can lead to great fluctuations of the arc voltage even though the cathode root does not move.

In this work we show the ST ratio to be a critical parameter in describing the splitter plate performance. It is shown that the arc remains in the splitter plates for longer using the ST ratio, under the wider vent condition because the wider vent provides better conditions for gas outflow from the quenching chamber and the arc moves further and more quickly to the splitter plates. In addition, the well distributed vent gives more stable arcing in the splitter plate region with decreased arc back-steps, leading to improved switching performance. It can be explained by gas flow depending on vent distribution. Figure 16 shows a comparison of gas flows between the 2 and 12 apertures vents. In the 12 apertures case, the gas can uniformly flow out of the chamber and there are no regions for gas flow that are blocked (see Fig. 16 (b)). However, in 2 apertures vent, the gas mainly flows through two paths (the upper and bottom path) in the splitter plate region as shown in Fig. 16 (a), and there is a relatively high pressure region between two apertures (A in Fig. 16 (a)), which leads to blocking effect on gas flow. This blocking effect deteriorates gas flow inside the quenching chamber. Moreover, it increases the hot gas staying in front of the splitter plates region instead of flowing out through vents, which can result in the formation of new arc path in front of the splitter plates.

\section{CONCLUSION}

In this paper, an experimental investigation has been carried out regarding arc motion near splitter plates in LVSDs. The following conclusions can be drawn.

1) The high frequency fluctuation of arc voltage during the switching process is significantly dependent on instability of arc motion (arc roots and column position) near the splitter plates; when the arc moves further into plates the arc voltage increases, whereas the voltage drops when the arc retreats away from plates.

2) Before the arc reaches the splitter plates, the arc voltage is highly correlated to the cathode root trajectory; however, inside the splitter plates there can be a high fluctuation voltage for a given arc root position.

3) The $S T$ ratio is a critical parameter in describing the splitter plate performance. The wider vent condition contributes the higher ST ratio of the arc in the splitter plate region since the arc moves further and more quickly due to the better conditions for gas outflow through the wider vent.

4) The well distributed vent achieves a higher ST ratio (arc's further movement and more stable staying in the splitter plate region) with decreased arc back-steps because the gas uniformly flows out through well distributed opening area with less residual hot gas left in front of the splitter plates (near the splitter plates).

\section{REFERENCES}

[1] E. Belbel and M. Lauraire, "Behavior of switching arc in low-voltage limiter circuit breakers," IEEE Trans. Components, Hybrids, Manuf. Technol., vol. 8, no. 1, pp. 3-12, Mar. 1985.

[2] D. Shin, I. O. Golosnoy, and J. W. McBride, "Experimental study of re-ignition evaluators in low voltage switching devices," IEEE Trans. Components Packag. Manuf. Technol., vol. 8, no. 6, PP. 950-957, Mar. 2018.

[3] J. W. McBride, K. Pechrach, and P. M. Weaver, "Arc root commutation from moving contacts in low voltage devices," IEEE Trans. Components Packag. Technol., vol. 24, no. 3, pp. 331-336, Sep. 2001.

[4] J. W. McBride, K. Pechrach, and P. M. Weaver, "Arc motion and gas flow in current limiting circuit breakers operating with a low contact switching velocity," IEEE Trans. Components Packag. Technol., vol. 25, no. 3, pp. 427-433, Sep. 2002.

[5] J. W. McBride, P. M. Weaver, and P. A. Jeffery, "Arc root mobility during contact opening at high current," IEEE Trans. components, Packag. Manuf. Technol. Part A, vol. 21, no. 1, pp. 61-67, Mar. 1998.

[6] X. Li, D. Chen, R. Dai, and Y. Geng, "Study of the influence of arc ignition position on arc motion in low-voltage circuit breaker," IEEE Trans. Plasma Sci., vol. 35, no. 2, pp. 491-497, Apr. 2007.

[7] Y. Wu, M. Rong, X. Li, A. B. Murphy, X. Wang, F. Yang, and Z. Sun, "Numerical analysis of the effect of the chamber width and outlet area on the motion of an air arc plasma," IEEE Trans. Plasma Sci., vol. 36, no. 5, pp. 2831-2837, Oct. 2008.

[8] D. Shin, I. O. Golosnoy, T. G. Bull, and J. W. McBride, "Experimental study on the influence of vent aperture size and distribution on arc motion and interruption in low-voltage switching devices," 2017 4th International Conference on Electric Power Equipment - Switching Technology, 2017, pp. 213-217.

[9] D. Chen, R. Dai, and X. Li, "Experimental investigation on the arc motion with different configurations of quenching chamber in AC contactor," IEICE Trans. Electron., vol. E89-C, no. 8, pp. 1201-1205, Aug. 2006.

[10] W. Hauer, "Re-ignition phenomena in low-voltage circuit breakers," Ph.D. dissertation, Vienna University of Technology, Austria, 2012.

[11] M. Lindmayer, E. Marzahn, A. Mutzke, T. Rüther, and M. Springstubbe, "The process of arc splitting between metal plates in low voltage arc chutes," IEEE Trans. Components Packag. Technol., vol. 29, no. 2, pp. 310-317, Jun. 2006.

[12] J. W. McBride, D. Shin and T. Bull, "A study of the motion of high current arcs in splitter plates using an arc imaging system," in Proc. ICEC, 2016, pp. 175-180.

[13] J. W. Mcbride, A. Balestrero, L. Ghezzi, G. Tribulato, and K. J. Cross, "Optical fiber imaging for high speed plasma motion diagnostics: Applied to low voltage circuit breakers," Review of Scientific Instruments 81, 055109, 2010, DOI: 10.1063/1.3428737.

[14] D. Shin, T. G. Bull, and J. W. McBride, "Experimental Study of Relationship Between Arc Light Intensity and Temperature in Low-Voltage Switching Devices," IEEE Trans. Components Packag. Manuf. Technol., vol. 10, no. 11, PP. 1840-1848, Nov. 2020.

[15] D. Shin, J. W. McBride, and I. O. Golosnoy, "Arc modelling to predict arc extinction in low-voltage switching devices," in Proc. 64th IEEE Holm Conf. Elect. Contacts, USA, Oct. 2018. 